\title{
Technical note: Comparative evaluation of 3 methods for the quantification of nonesterified fatty acids in bovine plasma sampled prepartum
}

\author{
Angel Abuelo,* ๑ Jill L. Brester, Kathryn Starken, and Louis M. Neuder \\ Department of Large Animal Clinical Sciences, College of Veterinary Medicine, Michigan State University, East Lansing 48824
}

\begin{abstract}
Serum or plasma concentrations of nonesterified fatty acids (NEFA) are commonly used as biomarkers of lipolysis during the periparturient period in dairy cows. However, NEFA quantification usually requires sample submission to specialized diagnostic laboratories, at significant cost. Alternative methods for the measurement of NEFA concentrations are needed that decrease the cost per sample without compromising accuracy and precision. Our study compared the quantification of NEFA between the gold standard diagnostic laboratory method and 2 alternative methods: a 96-well plate protocol and a small-scale chemistry analyzer (CataChemWell-T; Catachem Inc., Oxford, CT). We used a total of 147 plasma samples collected from cows 7 to $13 \mathrm{~d}$ before their expected calving date $(7 \pm 3.3$; mean $\pm \mathrm{SD}$ days before actual calving $)$ were used. We used linear and Passing-Bablok regression to identify systematic and proportional bias between the alternative methods and the gold standard. We also examined the level of agreement between each alternative method and the gold standard using Bland-Altman plots. We calculated the sensitivity and specificity of the alternative methods for detecting animals with excessive lipid mobilization prepartum (defined as NEFA concentration $\geq 0.30 \mathrm{~m} M$ by the gold standard test). We identified a constant difference between each of the alternative NEFA determination methods and the gold standard. Nevertheless, the mean bias was relatively small $(-0.03 \mathrm{mM}$ and $-0.02 \mathrm{~m} M$ for the 96 -well plate and small-scale analyzer methods, respectively). However, this tendency to underestimate NEFA concentrations had only a minimal effect on the ability of the tests to detect cows with excessive lipid mobilization prepartum (specificity 100\%; sensitivity 88.9 and $94.4 \%$ for the 96-well plate and small-scale analyzer methods, respectively). The 96-well plate and small-scale chem-
\end{abstract}

Received August 30, 2019.

Accepted November 24, 2019.

*Corresponding author: abuelo@msu.edu istry analyzer methods tested in this study are suitable for the quantification of NEFA concentrations in plasma and the dichotomous classification of samples as indicators of excessive prepartum lipid mobilization. Key words: dairy cow, energy deficit, fat mobilization, metabolic health indicators

\section{Technical Note}

Dairy cows experience metabolic stress during the periparturient period when they fail to adapt physiologically to the profound increase in nutrient requirements associated with fetal growth and milk production (Sordillo and Mavangira, 2014). Metabolic stress is characterized by excessive lipid mobilization, oxidative stress, and inflammatory dysfunction (Abuelo et al., 2015), and it has negative effects on both the cow and its offspring (Abuelo et al., 2019). Serum or plasma concentrations of nonesterified fatty acids (NEFA) and BHB are commonly used as biomarkers of energy deficit during the periparturient period (Ospina et al., 2010a,c; McArt et al., 2013; Ospina et al., 2013). Concentrations of these biomarkers above established cutoff points during the pre- and postpartum periods have been associated with increased risk of clinical disease (Ospina et al., 2010a; Chapinal et al., 2011; McArt et al., 2013), decreased milk production (Duffield et al., 2009; Ospina et al., 2010c; Chapinal et al., 2012), decreased reproductive success (Ospina et al., 2010c; Chapinal et al., 2012; Garverick et al., 2013), and increased risk of being removed from the herd (Ospina et al., 2010c; McArt et al., 2012; Roberts et al., 2012).

Although NEFA and BHB are related to energy deficit, they cannot be used interchangeably because these biomarkers are not very well associated when sampled at the same time point (Ospina et al., 2013), or over time across the periparturient period (McCarthy et al., 2015). This weak association between NEFA and BHB concentrations might be attributed to ketone synthesis depending on energy deficit (i.e., lipolysis), the availability of intermediates in the tricarboxylic acid cycle, and individual differences in hepatic gluconeogenic capacity (McCarthy et al., 2015). Elevations 
of NEFA concentrations above established thresholds have consistently reported better ability to predict the risk of negative downstream outcomes during both the pre- and postpartum periods than BHB concentrations (Ospina et al., 2010a,b; McArt et al., 2013). However, in fresh cows, BHB quantification is preferred for monitoring because several point-of-care devices have been validated for cow-side use (Iwersen et al., 2009, 2013; Bach et al., 2016) or in stored samples (Leal Yepes et al., 2018), whereas NEFA determination requires the submission of samples to a specialized laboratory (Abuelo and Alves-Nores, 2016).

At the Michigan State University Veterinary Diagnostic Laboratory (MSU-VDL; East Lansing MI), each sample analyzed for NEFA concentration using the gold standard wet chemistry method costs $\$ 17$ (as of August 2019). Alternative methods are needed for the measurement of NEFA concentrations that decrease the cost per sample without compromising the accuracy and precision. Early studies dealt with the modification of the validated commercial kit to accommodate smaller sample volumes, reduce assay cost, and increase sample throughput in humans (Jeevanandam et al., 1989; Christmass et al., 1998). In cattle, Johnson and Peters (1993) reported good recovery, reagent stability, and range of linearity of NEFA quantification using a 96-well plate format; and Irina et al. (2004) compared pairs of measurements for bovine serum samples using a mean difference plot, concluding that despite some bias in the measurement, the 96-well plate format could be used reliably. However, to our knowledge, the ability of the 96-well plate format to detect cows experiencing excessive lipid mobilization (NEFA concentration $\geq 0.30 \mathrm{~m} M$ ) remains unexplored. In addition, small-scale chemistry analyzers are now commercially available and provide another costeffective alternative for the quantification of NEFA for research or herd monitoring. Our objective was to compare the accuracy and diagnostic utility of plasma NEFA determinations using a 96-well plate method and a semi-automatic small-scale biochemistry analyzer (CataChemWell-T; Catachem Inc., Oxford, CT) with the gold standard determination at the MSU-VDL [HR Series NEFA-HR(2); Fujifilm Wako Diagnostics USA Corporation, Mountain View, CA]. Our hypothesis was that the results of both methods would be comparable to the gold standard test.

Our study was conducted on a large commercial dairy farm associated with the Michigan State University Training Center for Dairy Professionals (Elsie, MI) from February to August 2019. The farm has an average of 3,500 lactating cows, with a rolling herd average milk production of $12,250 \mathrm{~kg} / \mathrm{cow}$. Exemption from protocol review was granted by the Michigan State University Institutional Animal Care and Use Committee because we used only samples collected as part of the farm's routine herd monitoring program. In brief, blood was collected via puncture of the coccygeal vessels using EDTA vacuum tubes (Monoject EDTA K3; Covidien, Minneapolis, MN) from a random sample of 10 close-up cows every 2 wk. All multiparous cows expected to calve 7 to $13 \mathrm{~d}$ after the sampling date were eligible for enrolment, and 10 cows were selected from this group using randomization software (www .graphpad.com/quickcalcs/randomSelect1/). We used a total of 150 samples for our study. After blood collection, samples were immediately centrifuged on the farm at 2,000 $\times g$ for $10 \mathrm{~min}$, and the plasma was harvested and aliquoted into 2-mL microcentrifuge tubes (Fisher Scientific, Waltham, MA). One aliquot was submitted to CentralStar Cooperative (Grand Ledge, MI) for NEFA quantification using the 96-well plate adapted protocol, and another was transported to our laboratory for NEFA determination using a small-scale semi-automatic analyzer and subsequent submission to MSU-VDL. All aliquots were handled under refrigeration pending analysis within $24 \mathrm{~h}$ after collection.

In the MSU-VDL, aliquots were analyzed for NEFA concentration using an autoanalyzer (AU 680 series; Beckman Coulter Inc., Brea, CA), and a commercial validated assay [HR Series NEFA-HR(2); Fujifilm Wako Diagnostics USA Corp.]. The assay was calibrated using commercial control sera (Control Serum II; Fujifilm Wako Diagnostics USA Corp.) every $10 \mathrm{~d}$ or after changes in reagents or equipment. The commercial assay is based in the enzymatic Acyl-CoA oxidase method (Matsubara et al., 1983). At CentralStar Cooperative, aliquots were analyzed using the same commercial reagents as at MSU-VDL, but following a protocol adapted for 96-well plates, as described by Johnson and Peters (1993). A 5-point standard curve $(0,0.125,0.25,0.5,1.0 \mathrm{mM})$ of a nonesterified oleic acid standard (NEFA Standard; Fujifilm Wako Diagnostics USA Corp.) was fitted into every plate. Samples were analyzed in duplicate, and those with readings outside of the standard curve were diluted 1:2 with PBS and reassayed. The intra- and inter-assay CVs were 6.6 and $7.7 \%$ for the 96 -well plate method, and ranged from 1.1 to $2.9 \%$ and 2.1 to $11.5 \%$, respectively, for the MSUVDL method.

In our laboratory, the concentration of NEFA in the samples was determined using a small-scale biochemistry analyzer (CataChemWell-T; Catachem Inc.) and reagents from the same manufacturer (NEFA; Catachem Inc.) that were also based on the Acyl-CoA oxidase method. The analyzer was calibrated every 2 wk using 
Table 1. Analytical precision of nonesterified fatty acid (NEFA) quantification using a small-scale biochemistry analyzer $^{1}$

\begin{tabular}{|c|c|c|c|c|c|c|}
\hline \multirow[b]{3}{*}{ Sample } & \multicolumn{6}{|c|}{ Precision $^{2}$} \\
\hline & \multicolumn{3}{|c|}{ Intra-assay } & \multicolumn{3}{|c|}{ Inter-assay } \\
\hline & Mean $(\mathrm{m} M)$ & $\mathrm{SD}$ & CV $(\%)$ & Mean $(\mathrm{m} M)$ & $\mathrm{SD}$ & CV $(\%)$ \\
\hline 1 & 0.50 & 0.014 & 2.9 & 0.55 & 0.043 & 7.8 \\
\hline 2 & 0.19 & 0.006 & 3.3 & 0.20 & 0.018 & 9.3 \\
\hline 3 & 0.14 & 0.005 & 3.5 & 0.14 & 0.012 & 8.3 \\
\hline 4 & 0.06 & 0.005 & 9.0 & - & - & - \\
\hline
\end{tabular}

a commercial calibrator (NEFA Calibrator; Catachem Inc.) and 2-level control material (NEFA Control Level I and Control Level II; Catachem Inc.). We determined the precision of this method following the American Society for Veterinary Clinical Pathology Quality Assurance and Laboratory Standard Guidelines (GunnChristie et al., 2012). Intra-assay CVs were determined on 20 replications of 4 samples and inter-assay CVs were determined for 15 consecutive days for 3 samples (Table 1).

We compared methods following the American Society for Veterinary Clinical Pathology guidelines (Jensen and Kjelgaard-Hansen, 2006). Data analysis was performed in JMP Pro 14.0 (SAS Institute Inc., Cary, NC). The normality of data was assessed with the Shapiro-Wilk test. Spearman correlation coefficients between the NEFA gold standard and NEFA concentrations obtained using the 96-well plate and smallscale analyzer methods. Regression analysis between each method and the gold standard was performed using JMP Pro 14.0. Linearity between methods was visually assessed using the plot of residuals. PassingBablok regression coefficients, slopes, and intercepts were obtained using MedCalc Statistical Software (version 19.0.7; Med-Calc Software, Ostend, Belgium). This regression fits a straight line to 2 variables and is less sensitive to outliers because it assumes measurement errors in both methods. Perfect agreement yields an intercept of 0 and a slope of 1 . We generated BlandAltman plots with GraphPad Prism (v. 8; GraphPad Software, La Jolla, CA) to graphically demonstrate the level of agreement between 2 tests that cannot be captured by the correlation estimate alone (Bland and Altman, 1986). The Bland-Altman plot includes a solid horizontal line showing the mean bias (mean value of differences) between the 2 methods, as well as the $95 \%$ confidence interval (95\% CI) of agreement. Sensitivity of each method was calculated as the proportion of animals properly diagnosed with excessive lipid mobilization [NEFA plasma concentration $\geq 0.30 \mathrm{mM}$; Ospina et al. (2010a)] among all animals identified as positive by each method compared with those classified as positive using the gold standard test (NEFA determination at MSU-VDL). We calculated specificity as the proportion of animals properly diagnosed as negative (NEFA plasma concentration $<0.30 \mathrm{mM}$ ) among all animals identified as negative by each method compared with those classified as negative using the gold standard. We calculated the $95 \%$ CI for sensitivity and specificity values in JMP Pro 14.0.

To reduce the effect of hemolysis on NEFA quantification (Stokol and Nydam, 2006), we excluded 3 of the 150 plasma samples collected due to moderate or high hemolysis indices reported by MSU-VDL. Of the remaining 147 samples, 18 had a NEFA concentration $\geq 0.30 \mathrm{~m} M$ as assessed using the gold standard test. Data distribution of NEFA analyzed via the gold standard test showed a nonparametric distribution $(P$ $<0.001$ ). The median was $0.13 \mathrm{mM}$, and the range was 0.03 to $2.42 \mathrm{~m} M$. Spearman correlation coefficients between the gold standard test and the 96-well plate and small-scale analyzer methods were 0.98 and 0.99 , respectively.

Linear regression analysis revealed a linear relationship between both alternative methods and the gold standard test $\left(R^{2}=0.97\right.$ and 0.98 for the 96 -well plate and small-scale analyzer methods, respectively). However, graphical visualization of residuals revealed a deviation from normality in both alternative methods, suggesting a lack of fit of the linear regression models. Linear regression is commonly used when comparing 2 methods with continuous outcomes, but it assumes normality of the data distribution and absence of error in the gold standard test. Therefore, we explored Passing-Bablok regression because it allowed us to compare methods with data that had nonparametric distribution 
(Jensen and Kjelgaard-Hansen, 2006). The intercept indicates the systematic bias between the 2 methods, and the slope is interpreted as proportional bias between the 2 methods. The Spearman rank correlation coefficients from Passing-Bablok regressions showed a strong correlation $(>0.90)$ between the alternative methods and the gold standard assay. Both alternative methods showed an intercept slightly different from zero (Figure 1), indicating a tendency to systematically underesti-
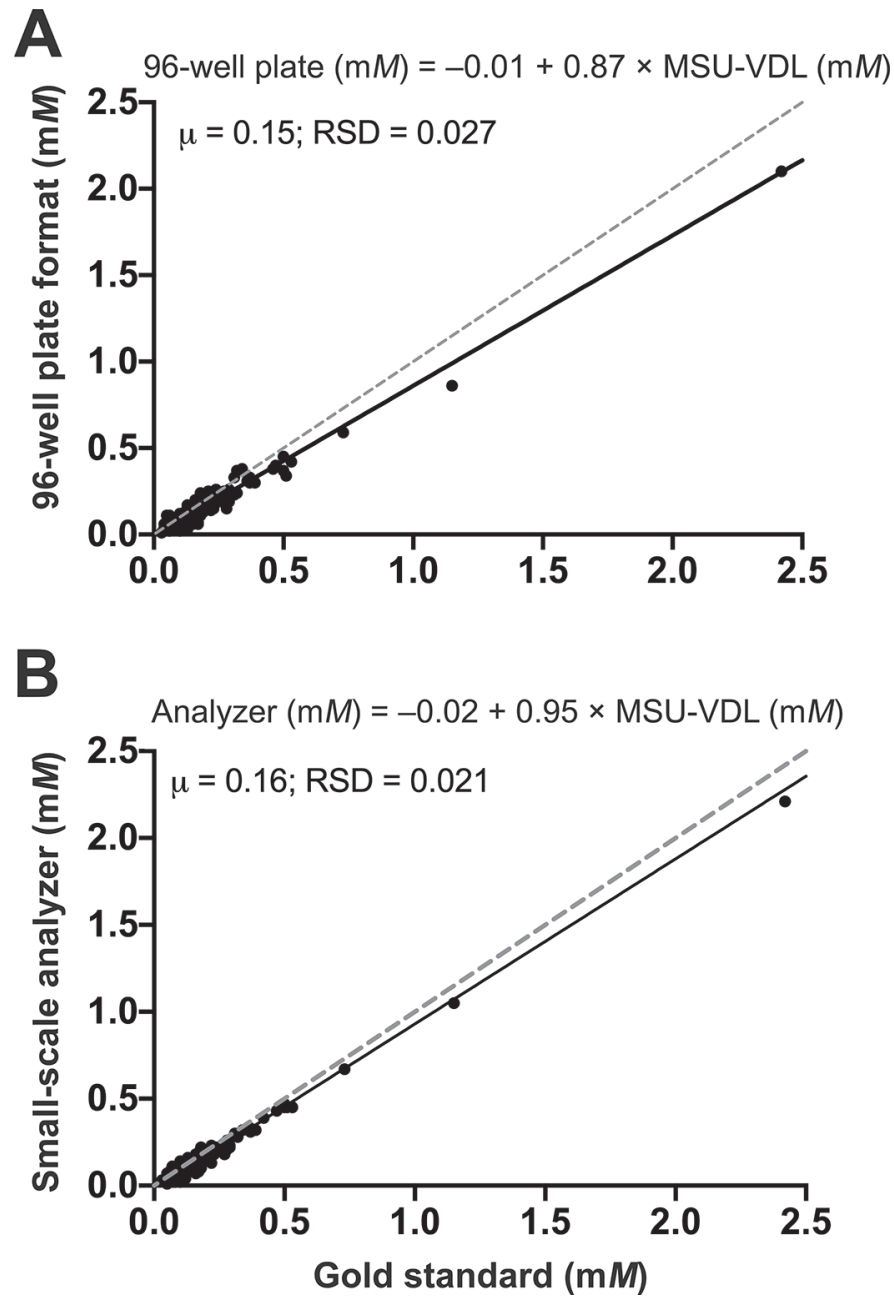

Figure 1. Passing-Bablok regression analysis between nonesterified fatty acid (NEFA) plasma concentrations measured using the gold standard test (Fujifilm Wako Diagnostics USA Corp., Mountain View, CA) at the Michigan State University Veterinary Diagnostic Laboratory and (A) a protocol adapted to use in a 96-well plate format using the same reagents, or (B) a small-scale analyzer and commercial reagents from the same brand (Catachem Inc., Oxford, CT). A total of 147 samples collected from cows 7 to $13 \mathrm{~d}$ before their expected calving date ( $7 \pm 3.3 \mathrm{~d}$; mean \pm SD before actual calving) were used. The gray dashed line is the line of identity $(\mathrm{Y}=\mathrm{X})$ and represents perfect agreement between the 2 methods. $\mu=$ the mean of all NEFA concentrations measured by each method; RSD = residual standard deviation.
Table 2. Passing-Bablok regression coefficients for nonesterified fatty acid (NEFA) concentrations in 147 prepartum $^{1}$ plasma samples measured with 2 alternative methods compared with the gold standard test $^{2}$

\begin{tabular}{lll}
\hline Method & Intercept $(95 \%$ CI $)$ & Slope $(95 \%$ CI $)$ \\
\hline 96-well plate format & $-0.01(-0.02,-0.01)$ & $0.87(0.81,0.93)$ \\
Small-scale analyzer & $-0.02(-0.03,-0.01)$ & $0.95(0.92,1.00)$ \\
\hline
\end{tabular}

${ }^{1}$ Samples were collected 7 to $13 \mathrm{~d}$ before the expected calving date $(7$ $\pm 3.3 \mathrm{~d}$; mean $\pm \mathrm{SD}$ before actual calving).

${ }^{2}$ Only the gold standard method was performed at the Michigan State University Veterinary Diagnostic Laboratory (East Lansing, MI).

mate the concentration of NEFA compared to the gold standard. The $95 \%$ CI of the intercept did not include zero for either of the alternative methods (Table 2), indicating the presence of constant bias between each of the alternative methods and the gold standard (Passing and Bablok, 1983).

The test agreement between NEFA plasma concentrations measured by the gold standard and both alternative methods is illustrated in Figure 2. The 96-well plate method had a mean bias (i.e., the average of the differences in accuracy of the tested method compared with the gold standard) of $-0.03 \mathrm{mM}$ (95\% CI -0.13 to 0.06 ), and the small-scale analyzer had a mean bias of $-0.02 \mathrm{mM}$ (95\% CI -0.09 to 0.04$)$ compared to the gold standard test. The mean bias was negative for both methods, meaning that NEFA concentrations were generally underestimated in these methods. The mean bias we determined for the 96 -well plate method was smaller than the $0.06 \mathrm{~m} M(95 \%$ CI -0.10 to 0.13$)$ reported by Irina et al. (2004) when comparing the 96-well plate method to the gold standard test using bovine serum samples. Interestingly, Irina et al. (2004) reported a positive bias with the 96 -well plate method, whereas we found a negative bias. However, the CI were similar and included zero in both studies, indicating a similar bias pattern. The small-scale analyzer showed a smaller bias than the 96 -well plate method, as determined by the smaller mean bias and the narrower $95 \%$ confidence limits of agreement. Nevertheless, the biases of both methods were relatively low, suggesting that both methods were acceptable alternatives to the gold standard for quantifying NEFA in plasma samples. However, users of these methods should be aware of this bias, because it could interfere with the interpretation of results, especially for values close to established cutoff points or for samples with high NEFA concentrations, where we observed greater differences (Figure 2). Similarly, this bias needs to be considered when computing and comparing quantitative NEFA concentrations for research purposes. 

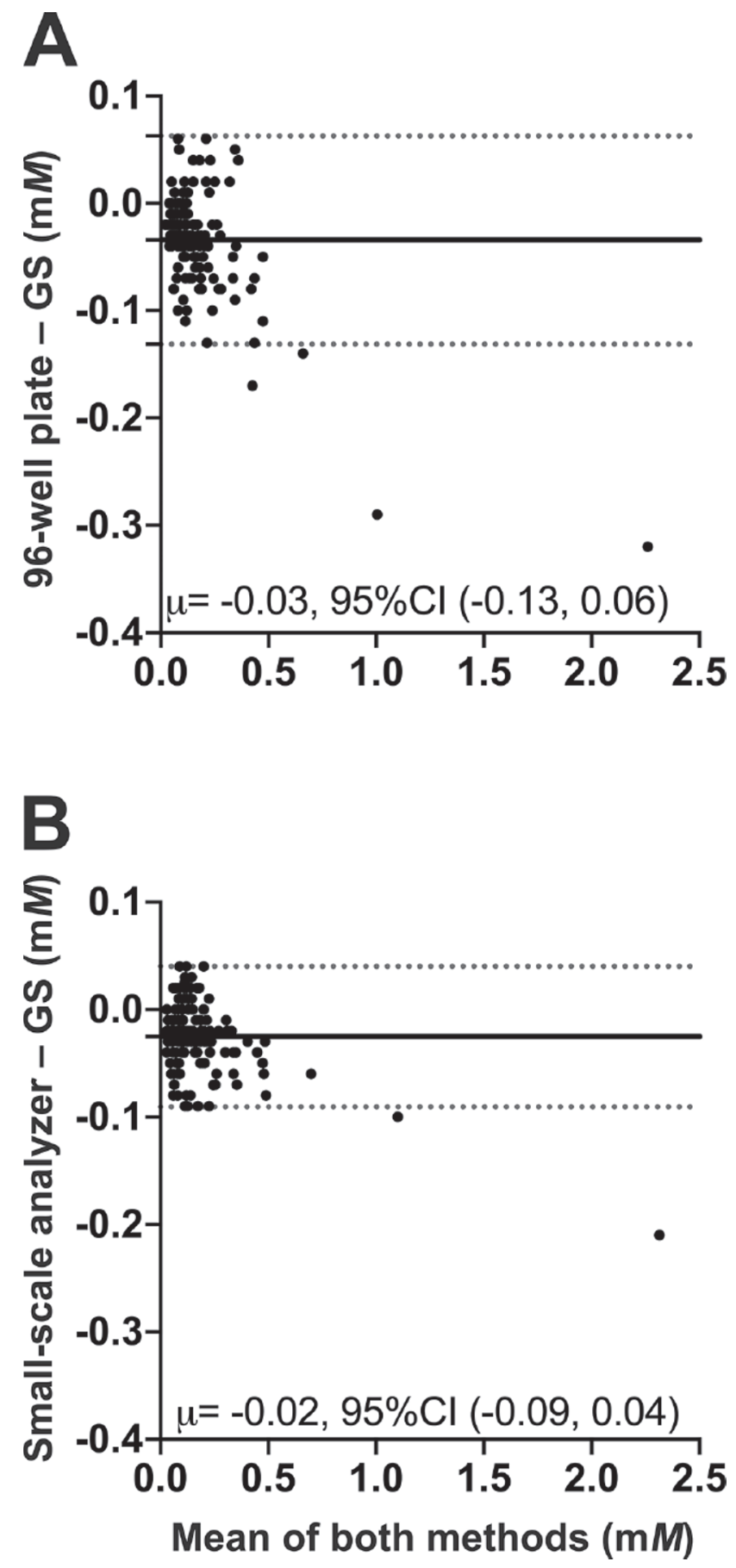

Figure 2. Bland-Altman plots of the difference in nonesterified fatty acid (NEFA) plasma concentrations between the 2 tests against their mean: (A) 96-well plate format versus the gold standard (GS) test at the Michigan State University Veterinary Diagnostic Laboratory (MSU-VDL); and (B) small-scale analyzer versus the GS test. The solid horizontal line represents the mean bias; horizontal dashed lines represent the $95 \%$ CI of agreement. A negative mean bias shows an underestimation of the NEFA concentration by the alternative method compared with the gold standard test. A total of 147 samples collected from cows 7 to $13 \mathrm{~d}$ before their expected calving date $(7 \pm 3.3 \mathrm{~d}$; mean \pm SD before actual calving) were used. $\mu=$ the overall mean bias $(\mathrm{mM})$ calculated as the alternative method (either 96-well plate or small-scale analyzer) minus the GS value.
To study the effect of this bias on the correct classification of excessive lipid mobilization prepartum in dairy cows, we calculated the sensitivity and specificity of both alternative methods compared with the gold standard test (Table 3). Both the 96-well plate method and the small-scale analyzer had perfect specificity for excessive lipid mobilization prepartum. The sensitivity was slightly higher for the small-scale analyzer than for the 96 -well plate method (94.4 vs. $88.9 \%$ ), although both were considered good. The lower sensitivity of the alternative methods is likely because of the underestimation of NEFA concentration (negative mean bias), resulting in incorrect classification of some cows with NEFA concentrations slightly above the $0.30 \mathrm{~m} M$ cutoff. Users of these methods should be aware of this underestimation bias and carefully interpret results that are slightly below established cutoff points for NEFA concentrations. To our knowledge, this is the first study to investigate the diagnostic ability of different methods of NEFA quantification, so the values obtained could not be compared with existing literature. Based on the sensitivity and specificity results, the tested alternative methods for NEFA quantification can be used with satisfactory diagnostic utility to identify cows experiencing excessive lipid mobilization pre-calving.

The 96-well plate and small-scale chemistry analyzer methods tested in this study are suitable for the quantitative determination of NEFA concentrations in plasma and the dichotomous classification of samples as indicators of excessive prepartum lipid mobilization. Our findings enable the use of these alternative methods for herd monitoring and research purposes at a lower cost per sample.

Table 3. Performance of 2 alternative methods of nonesterified fatty acid (NEFA) quantification for the classification of excessive lipid mobilization $^{1}$ precalving $^{2}$

\begin{tabular}{lcc}
\hline Method & $\begin{array}{c}\text { Sensitivity, }{ }^{3} \% \\
(95 \% \text { CI })\end{array}$ & $\begin{array}{c}\text { Specificity, } \\
(95 \% \text { CI })\end{array}$ \\
\hline 96-well plate format & $88.9(67.2,96.9)$ & $100.0(97.1,100.0)$ \\
Small-scale analyzer & $94.4(74.2,99.0)$ & $100.0(97.1,100.0)$ \\
\hline
\end{tabular}

${ }^{1}$ Defined as NEFA plasma concentration $\geq 0.30 \mathrm{~m} M$ as measured by the gold standard test.

${ }^{2} \mathrm{~A}$ total of 147 plasma samples collected from cows 7 to $13 \mathrm{~d}$ before their expected calving date $(7 \pm 3.3 \mathrm{~d}$; mean $\pm \mathrm{SD}$ before actual calving) were used; 18 of those had NEFA concentrations $\geq 0.30 \mathrm{mM}$ as measured by the gold standard test.

${ }^{3}$ Sensitivity was calculated as the proportion of animals classified as positive for excessive lipid mobilization prepartum compared with the animals classified as positive using the gold standard test (NEFA concentration $\geq 0.30 \mathrm{~m} M$ ) performed at the Michigan State University Veterinary Diagnostic Laboratory (MSU-VDL, East Lansing, MI).

${ }^{4}$ Specificity was calculated as the proportion of animals classified as negative for excessive lipid mobilization prepartum compared with animals classified as negative using the gold standard test (NEFA concentration $<0.30 \mathrm{mM}$ ) performed at the MSU-VDL. 


\section{ACKNOWLEDGMENTS}

This work was supported by the Animal Health Project 1016161 and the Agriculture and Food Research Initiative Competitive Grant no. 2018-67015-28302 from the USDA National Institute of Food and Agriculture (Washington, DC), as well as the AA19-02 grant of the Michigan Alliance for Animal Agriculture (East Lansing, MI). The authors thank Todd Byron (CentralStart, Grand Ledge, MI) for assistance with the 96-well plate NEFA determinations, and Jennifer Thomas, Marion Payne-Bushey, and Tom Herdt (Michigan State University Veterinary Diagnostic Laboratory, East Lansing, MI) for assistance with the gold standard NEFA analyses. The authors have not stated any conflicts of interest.

\section{REFERENCES}

Abuelo, A., and V. Alves-Nores. 2016. Point-of-care testing in cattle practice: Reliability of cow-side diagnostic tests. In Pract. 38:293302. https://doi.org/10.1136/inp.i2704.

Abuelo, A., J. Hernandez, J. L. Benedito, and C. Castillo. 2015. The importance of the oxidative status of dairy cattle in the periparturient period: Revisiting antioxidant supplementation. J. Anim. Physiol. Anim. Nutr. (Berl.) 99:1003-1016. https://doi.org/10 $.1111 /$ jpn. 12273 .

Abuelo, A., J. Hernandez, J. L. Benedito, and C. Castillo. 2019. Redox biology in transition periods of dairy cattle: Role in the health of periparturient and neonatal animals. Antioxidants 8:20. https:// doi.org/10.3390/antiox8010020.

Bach, K. D., W. Heuwieser, and J. A. A. McArt. 2016. Technical note: Comparison of 4 electronic handheld meters for diagnosing hyperketonemia in dairy cows. J. Dairy Sci. 99:9136-9142. https://doi .org/10.3168/jds.2016-11077.

Bland, J. M., and D. G. Altman. 1986. Statistical methods for assessing agreement between two methods of clinical measurement. Lancet 327:307-310. https://doi.org/10.1016/S0140-6736(86)90837-8.

Chapinal, N., M. Carson, T. F. Duffield, M. Capel, S. Godden, M. Overton, J. E. P. Santos, and S. J. LeBlanc. 2011. The association of serum metabolites with clinical disease during the transition period. J. Dairy Sci. 94:4897-4903. https://doi.org/10.3168/jds $.2010-4075$

Chapinal, N., M. E. Carson, S. J. LeBlanc, K. E. Leslie, S. Godden, M. Capel, J. E. Santos, M. W. Overton, and T. F. Duffield. 2012. The association of serum metabolites in the transition period with milk production and early-lactation reproductive performance. J. Dairy Sci. 95:1301-1309. https://doi.org/10.3168/jds.2011-4724.

Christmass, M. A., L. R. Mitoulas, P. E. Hartmann, and P. G. Arthur. 1998. A semiautomated enzymatic method for determination of nonesterified fatty acid concentration in milk and plasma. Lipids 33:1043-1049. https://doi.org/10.1007/s11745-998-0304-9.

Duffield, T. F., K. D. Lissemore, B. W. McBride, and K. E. Leslie. 2009. Impact of hyperketonemia in early lactation dairy cows on health and production. J. Dairy Sci. 92:571-580. https://doi.org/ $10.3168 /$ jds.2008-1507.

Garverick, H. A., M. N. Harris, R. Vogel-Bluel, J. D. Sampson, J. Bader, W. R. Lamberson, J. N. Spain, M. C. Lucy, and R. S. Youngquist. 2013. Concentrations of nonesterified fatty acids and glucose in blood of periparturient dairy cows are indicative of pregnancy success at first insemination. J. Dairy Sci. 96:181-188. https://doi.org/10.3168/jds.2012-5619.

Gunn-Christie, R. G., B. Flatland, K. R. Friedrichs, B. Szladovits, K. E. Harr, K. Ruotsalo, J. S. Knoll, H. L. Wamsley, and K. P.
Freeman. 2012. ASVCP quality assurance guidelines: Control of preanalytical, analytical, and postanalytical factors for urinalysis, cytology, and clinical chemistry in veterinary laboratories. Vet. Clin. Pathol. 41:18-26. https://doi.org/10.1111/j.1939-165X.2012 .00412.x.

Iwersen, M., U. Falkenberg, R. Voigtsberger, D. Forderung, and W. Heuwieser. 2009. Evaluation of an electronic cowside test to detect subclinical ketosis in dairy cows. J. Dairy Sci. 92:2618-2624. https: //doi.org/10.3168/jds.2008-1795.

Iwersen, M., D. Klein-Jobstl, M. Pichler, L. Roland, B. Fidlschuster, I. Schwendenwein, and M. Drillich. 2013. Comparison of 2 electronic cowside tests to detect subclinical ketosis in dairy cows and the influence of the temperature and type of blood sample on the test results. J. Dairy Sci. 96:7719-7730. https://doi.org/10.3168/ jds.2013-7121.

Jeevanandam, M., Y. C. Hsu, L. Ramias, and W. R. Schiller. 1989. A rapid, automated micromethod for measuring free fatty acids in plasma/serum. Clin. Chem. 35:2228-2231.

Jensen, A. L., and M. Kjelgaard-Hansen. 2006. Method comparison in the clinical laboratory. Vet. Clin. Pathol. 35:276-286. https://doi .org/10.1111/j.1939-165x.2006.tb00131.x.

Johnson, M. M., and J. P. Peters. 1993. Technical note: An improved method to quantify nonesterified fatty acids in bovine plasma. J. Anim. Sci. 71:753-756. https://doi.org/10.2527/1993.713753x.

Leal Yepes, F. A., D. V. Nydam, W. Heuwieser, and S. Mann. 2018. Technical note: Evaluation of the diagnostic accuracy of 2 pointof-care $\beta$-hydroxybutyrate devices in stored bovine plasma at room temperature and at $37^{\circ} \mathrm{C}$. J. Dairy Sci. 101:6455-6461. https://doi .org/10.3168/jds.2017-13960.

Matsubara, C., Y. Nishikawa, Y. Yoshida, and K. Takamura. 1983. A spectrophotometric method for the determination of free fatty acid in serum using acyl-coenzyme A synthetase and acyl-coenzyme A oxidase. Anal. Biochem. 130:128-133. https://doi.org/10.1016/ 0003-2697(83)90659-0.

McArt, J. A. A., D. V. Nydam, and G. R. Oetzel. 2012. Epidemiology of subclinical ketosis in early lactation dairy cattle. J. Dairy Sci. 95:5056-5066. https://doi.org/10.3168/jds.2012-5443.

McArt, J. A. A., D. V. Nydam, G. R. Oetzel, T. R. Overton, and P. A. Ospina. 2013. Elevated non-esterified fatty acids and betahydroxybutyrate and their association with transition dairy cow performance. Vet. J. 198:560-570. https://doi.org/10.1016/j.tvjl .2013.08.011.

McCarthy, M. M., S. Mann, D. V. Nydam, T. R. Overton, and J. A. McArt. 2015. Short communication: Concentrations of nonesterified fatty acids and beta-hydroxybutyrate in dairy cows are not well correlated during the transition period. J. Dairy Sci. 98:62846290. https://doi.org/10.3168/jds.2015-9446.

Irina, R. M., C. L. Buckley, and R. H. Poppenga. 2004. Detection of nonesterified (free) fatty acids in bovine serum: Comparative evaluation of two methods. J. Vet. Diagn. Invest. 16:139-144. https:// doi.org/10.1177/104063870401600208.

Ospina, P. A., J. A. McArt, T. R. Overton, T. Stokol, and D. V. Nydam. 2013. Using nonesterified fatty acids and beta-hydroxybutyrate concentrations during the transition period for herd-level monitoring of increased risk of disease and decreased reproductive and milking performance. Vet. Clin. North Am. Food Anim. Pract. 29:387-412. https://doi.org/10.1016/j.cvfa.2013.04.003.

Ospina, P. A., D. V. Nydam, T. Stokol, and T. R. Overton. 2010a. Evaluation of nonesterified fatty acids and beta-hydroxybutyrate in transition dairy cattle in the northeastern United States: Critical thresholds for prediction of clinical diseases. J. Dairy Sci. 93:546-554. https://doi.org/10.3168/jds.2009-2277.

Ospina, P. A., D. V. Nydam, T. Stokol, and T. R. Overton. 2010b. Associations of elevated nonesterified fatty acids and beta-hydroxybutyrate concentrations with early lactation reproductive performance and milk production in transition dairy cattle in the northeastern United States. J. Dairy Sci. 93:1596-1603. https:// doi.org/10.3168/jds.2009-2852.

Ospina, P. A., D. V. Nydam, T. Stokol, and T. R. Overton. 2010c. Association between the proportion of sampled transition cows 
with increased nonesterified fatty acids and $\beta$-hydroxybutyrate and disease incidence, pregnancy rate, and milk production at the herd level. J. Dairy Sci. 93:3595-3601. https://doi.org/10.3168/ jds.2010-3074.

Passing, H., and W. Bablok. 1983. A new biometrical procedure for testing the equality of measurements from two different analytical methods. Application of linear regression procedures for method comparison studies in clinical chemistry, Part I. J. Clin. Chem. Clin. Biochem. 21:709-720. https://doi.org/10.1515/cclm.1983.21 .11.709.

Roberts, T., N. Chapinal, S. J. Leblanc, D. F. Kelton, J. Dubuc, and T. F. Duffield. 2012. Metabolic parameters in transition cows as indicators for early-lactation culling risk. J. Dairy Sci. 95:30573063. https://doi.org/10.3168/jds.2011-4937.
Sordillo, L. M., and V. Mavangira. 2014. The nexus between nutrient metabolism, oxidative stress and inflammation in transition cows. Anim. Prod. Sci. 54:1204-1214. https://doi.org/10.1071/AN14503.

Stokol, T., and D. V. Nydam. 2006. Effect of hemolysis on nonesterified fatty acid and $\beta$-hydroxybutyrate concentrations in bovine blood. J. Vet. Diagn. Invest. 18:466-469. https://doi.org/10.1177/ 104063870601800507.

\section{ORCIDS}

Angel Abuelo @ https://orcid.org/0000-0001-9734-0148 